\title{
DATA MINING TECHNIQUES AND APPLICATIONS
}

Mrs. Bharati M. Ramageri, Lecturer

Modern Institute of Information Technology and Research,

Department of Computer Application, Yamunanagar, Nigdi

Pune, Maharashtra, India-411044.

\begin{abstract}
Data mining is a process which finds useful patterns from large amount of data. The paper discusses few of the data mining techniques, algorithms and some of the organizations which have adapted data mining technology to improve their businesses and found excellent results.
\end{abstract}

Keywords: Data mining Techniques; Data mining algorithms; Data mining applications.

\section{Overview of Data Mining}

The development of Information Technology has generated large amount of databases and huge data in various areas. The research in databases and information technology has given rise to an approach to store and manipulate this precious data for further decision making. Data mining is a process of extraction of useful information and patterns from huge data. It is also called as knowledge discovery process, knowledge mining from data, knowledge extraction or data /pattern analysis.

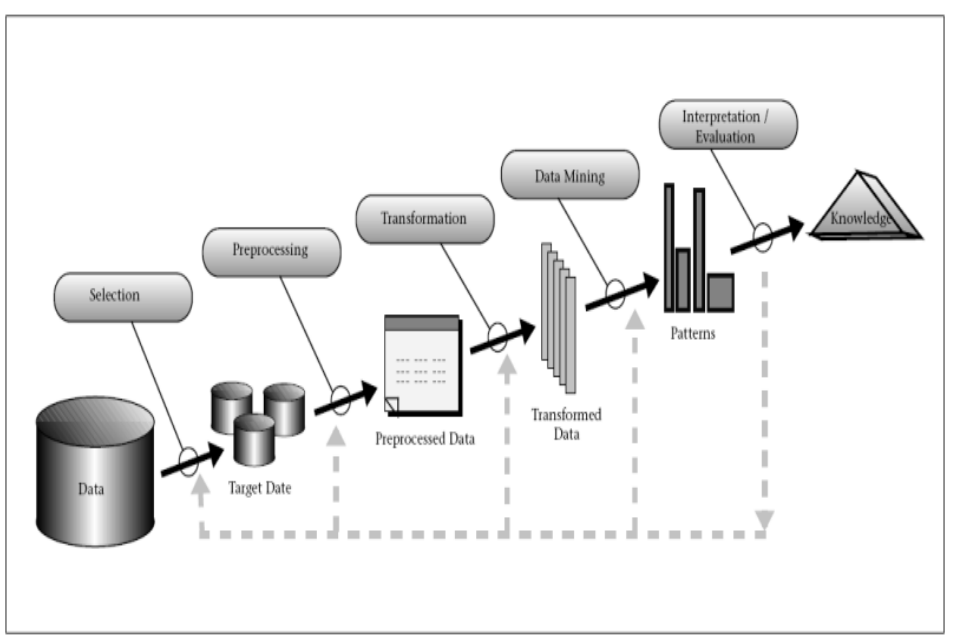

Figure 1. Knowledge discovery Process

Data mining is a logical process that is used to search through large amount of data in order to find useful data. The goal of this technique is to find patterns that were previously unknown. Once these patterns are found they can further be used to make certain decisions for development of their businesses.

Three steps involved are

- Exploration

- Pattern identification

- Deployment

Exploration: In the first step of data exploration data is cleaned and transformed into another form, and important variables and then nature of data based on the problem are determined. 
Pattern Identification: Once data is explored, refined and defined for the specific variables the second step is to form pattern identification. Identify and choose the patterns which make the best prediction.

Deployment: Patterns are deployed for desired outcome.

\section{Data Mining Algorithms and Techniques}

Various algorithms and techniques like Classification, Clustering, Regression, Artificial Intelligence, Neural Networks, Association Rules, Decision Trees, Genetic Algorithm, Nearest Neighbor method etc., are used for knowledge discovery from databases.

\subsection{Classification}

Classification is the most commonly applied data mining technique, which employs a set of pre-classified examples to develop a model that can classify the population of records at large. Fraud detection and creditrisk applications are particularly well suited to this type of analysis. This approach frequently employs decision tree or neural network-based classification algorithms. The data classification process involves learning and classification. In Learning the training data are analyzed by classification algorithm. In classification test data are used to estimate the accuracy of the classification rules. If the accuracy is acceptable the rules can be applied to the new data tuples. For a fraud detection application, this would include complete records of both fraudulent and valid activities determined on a record-by-record basis. The classifier-training algorithm uses these pre-classified examples to determine the set of parameters required for proper discrimination. The algorithm then encodes these parameters into a model called a classifier.

Types of classification models:

- Classification by decision tree induction

- Bayesian Classification

- Neural Networks

- $\quad$ Support Vector Machines (SVM)

- Classification Based on Associations

\subsection{Clustering}

Clustering can be said as identification of similar classes of objects. By using clustering techniques we can further identify dense and sparse regions in object space and can discover overall distribution pattern and correlations among data attributes. Classification approach can also be used for effective means of distinguishing groups or classes of object but it becomes costly so clustering can be used as preprocessing approach for attribute subset selection and classification. For example, to form group of customers based on purchasing patterns, to categories genes with similar functionality.

Types of clustering methods

- Partitioning Methods

- Hierarchical Agglomerative (divisive) methods

- Density based methods

- Grid-based methods

- $\quad$ Model-based methods 


\subsection{Predication}

Regression technique can be adapted for predication. Regression analysis can be used to model the relationship between one or more independent variables and dependent variables. In data mining independent variables are attributes already known and response variables are what we want to predict. Unfortunately, many real-world problems are not simply prediction. For instance, sales volumes, stock prices, and product failure rates are all very difficult to predict because they may depend on complex interactions of multiple predictor variables. Therefore, more complex techniques (e.g., logistic regression, decision trees, or neural nets) may be necessary to forecast future values. The same model types can often be used for both regression and classification. For example, the CART (Classification and Regression Trees) decision tree algorithm can be used to build both classification trees (to classify categorical response variables) and regression trees (to forecast continuous response variables). Neural networks too can create both classification and regression models.

Types of regression methods

- Linear Regression

- Multivariate Linear Regression

- Nonlinear Regression

- Multivariate Nonlinear Regression

\subsection{Association rule}

Association and correlation is usually to find frequent item set findings among large data sets. This type of finding helps businesses to make certain decisions, such as catalogue design, cross marketing and customer shopping behavior analysis. Association Rule algorithms need to be able to generate rules with confidence values less than one. However the number of possible Association Rules for a given dataset is generally very large and a high proportion of the rules are usually of little (if any) value.

\section{Types of association rule}

- Multilevel association rule

- Multidimensional association rule

- Quantitative association rule

\subsection{Neural networks}

Neural network is a set of connected input/output units and each connection has a weight present with it. During the learning phase, network learns by adjusting weights so as to be able to predict the correct class labels of the input tuples. Neural networks have the remarkable ability to derive meaning from complicated or imprecise data and can be used to extract patterns and detect trends that are too complex to be noticed by either humans or other computer techniques. These are well suited for continuous valued inputs and outputs. For example handwritten character reorganization, for training a computer to pronounce English text and many real world business problems and have already been successfully applied in many industries. Neural networks are best at identifying patterns or trends in data and well suited for prediction or forecasting needs.

Types of neural networks

- Back Propagation 


\section{Data Mining Applications}

Data mining is a relatively new technology that has not fully matured. Despite this, there are a number of industries that are already using it on a regular basis. Some of these organizations include retail stores, hospitals, banks, and insurance companies. Many of these organizations are combining data mining with such things as statistics, pattern recognition, and other important tools. Data mining can be used to find patterns and connections that would otherwise be difficult to find. This technology is popular with many businesses because it allows them to learn more about their customers and make smart marketing decisions. Here is overview of business problems and solutions found using data mining technology.

\subsection{FBTO Dutch Insurance Company}

Challenges

- $\quad$ To reduce direct mail costs.

- $\quad$ Increase efficiency of marketing campaigns.

- Increase cross-selling to existing customers, using inbound channels such as the company's sell center and the internet a one year test of the solution's effectiveness.

Results

- $\quad$ Provided the marketing team with the ability to predict the effectiveness of its campaigns.

- Increased the efficiency of marketing campaign creation, optimization, and execution.

- $\quad$ Decreased mailing costs by 35 percent.

- Increased conversion rates by 40 percent.

3.2. ECtel Ltd., Israel

Challenges

- $\quad$ Fraudulent activity in telecommunication services.

Results

- $\quad$ Significantly reduced telecommunications fraud for more than 150 telecommunication companies worldwide.

- $\quad$ Saved money by enabling real-time fraud detection.

\subsection{Provident Financial's Home credit Division, United Kingdom}

Challenges

- No system to detect and prevent fraud.

Results

- $\quad$ Reduced frequency and magnitude of agent and customer fraud.

- $\quad$ Saved money through early fraud detection.

- $\quad$ Saved investigator's time and increased prosecution rate.

\subsection{Standard Life Mutual Financial Services Companies}

Challenges

- Identify the key attributes of clients attracted to their mortgage offer.

- $\quad$ Cross sell Standard Life Bank products to the clients of other Standard Life companies.

- Develop a remortgage model which could be deployed on the group Web site to examine the profitability of the mortgage business being accepted by Standard Life Bank. 
Results

- $\quad$ Built a propensity model for the Standard Life Bank mortgage offer identifying key customer types that can be applied across the whole group prospect pool.

- $\quad$ Discovered the key drivers for purchasing a remortgage product.

- Achieved, with the model, a nine times greater response than that achieved by the control group.

- $\quad$ Secured £33million (approx. \$47 million) worth of mortgage application revenue.

\subsection{Shenandoah Life insurance company United States.}

Challenges

- $\quad$ Policy approval process was paper based and cumbersome.

- $\quad$ Routing of these paper copies to various departments, there was delays in approval.

Results

- $\quad$ Empowered management with current information on pending policies.

- $\quad$ Reduced the time required to issue certain policies by 20 percent.

- Improved underwriting and employee performance review processes.

\subsection{Soft map Company Ltd., Tokyo}

Challenges

- Customers had difficulty making hardware and software purchasing decisions, which was hindering online sales.

Results

- $\quad$ Page views increased 67 percent per month after the recommendation engine went live.

- $\quad$ Profits tripled in 2001, as sales increased 18 percent versus the same period in the previous year.

\section{Conclusion}

Data mining has importance regarding finding the patterns, forecasting, discovery of knowledge etc., in different business domains. Data mining techniques and algorithms such as classification, clustering etc., helps in finding the patterns to decide upon the future trends in businesses to grow. Data mining has wide application domain almost in every industry where the data is generated that's why data mining is considered one of the most important frontiers in database and information systems and one of the most promising interdisciplinary developments in Information Technology.

\section{References}

1. Jiawei Han and Micheline Kamber (2006), Data Mining Concepts and Techniques, published by Morgan Kauffman, 2nd ed.

2. Dr. Gary Parker, vol 7, 2004, Data Mining: Modules in emerging fields, CD-ROM.

3. Crisp-DM 1.0 Step by step Data Mining guide from http://www.crisp-dm.org/CRISPWP-0800.pdf.

4. Customer Successes in your industry from http://www.spss.com/success/?source=homepage\&hpzone=nav_bar.

5. https://www.allbusiness.com/Technology /computer-software-data-management/ 633425-1.html, last retrieved on 15th Aug 2010.

6. http://www.kdnuggets.com/. 